\title{
Expression of wingless-type mouse mammary tumor virus integration site family pathway effectors in lymphatic and hepatic metastases of patients with colorectal cancer: Associations with the primary tumor
}

\author{
SEBASTIAN KRAUS ${ }^{1}$, CHRISTIAN VAY ${ }^{1}$, STEPHAN BALDUS ${ }^{2}$, \\ WOLFRAM T. KNOEFEL ${ }^{1}$, NIKOLAS H. STOECKLEIN ${ }^{1}$ and DANIEL VALLBOHMER ${ }^{1}$ \\ ${ }^{1}$ Department of General, Visceral and Pediatric Surgery; ${ }^{2}$ Institute of Pathology, \\ University of Düsseldorf, Düsseldorf D-40225, Germany
}

Received May 8, 2014; Accepted February 5, 2015

DOI: $10.3892 / 01.2015 .3291$

\begin{abstract}
The wingless-type mouse mammary tumor virus integration site family (Wnt) pathway plays a major role in the carcinogenesis of colorectal cancer (CRC). Its most important effector, the nuclear $\beta$-catenin, influences not only transcription but also the proliferation and dedifferentiation of the colonic mucosa. This induces an epithelial-mesenchymal transition which ultimately can lead to the development of cancer and the formation of metastases. However, little is known about the exact interaction and context-sensitive expression of Wnt-pathway effectors in the primary tumor and corresponding metastasis. Therefore, this study assessed the expression of the three most important effectors of the Wnt pathway, $\beta$-catenin, adenomatous polyposis coli (APC) and Wnt-1, in the primary tumor and corresponding metastasis of patients with CRC. Immunohistochemical staining of $\beta$-catenin, APC and Wnt- 1 was performed in paraffin-embedded tissue samples of the primary tumor, and the corresponding hepatic and nodal metastasis samples from 24 patients with metastatic CRC. Isotype antibodies were used as negative controls. The results were visualized using the ABC-method. Analysis of the primary tumor comprised of a separate evaluation of the central tumor area as well as the invasion front. There was a significant overexpression of nuclear $\beta$-catenin at the tumor invasion front $(\mathrm{P}<0.001)$. Compared to normal colonic mucosa, expression of cytoplasmic $\beta$-catenin was significantly higher in the primary tumor $(\mathrm{P}<0.001)$ as well as all the corresponding hepatic and
\end{abstract}

Correspondence to: Professor Daniel Vallbohmer, Department of General, Visceral and Paediatric Surgery, University of Düsseldorf, 5 Moorenstrasse, Düsseldorf D-40225, Germany

E-mail: daniel.vallboehmer@med.uni-duesseldorf.de

Key words: colorectal cancer, wingless-type mouse mammary tumor virus integration site family pathway, primary tumor, distant metastases lymphatic metastases (hepatic metastases, $\mathrm{P}=0.001$; nodal metastases, $\mathrm{P}=0.017)$. By contrast, APC expression was significantly lower in all analyzed tumor compartments compared with normal colonic mucosa (primary tumor, $\mathrm{P}=0.022$; hepatic metastases, $\mathrm{P}=0.006$; nodal metastases, $\mathrm{P}=0.012$ ). Finally, Wnt-1 protein expression was significantly lower in liver metastases but not in the primary tumor or lymphatic metastases compared with normal colonic mucosa $(\mathrm{P}=0.003)$. The present study demonstrates that the major Wnt-effector proteins, $\beta$-catenin, APC and Wnt- 1 , are heterogeneously expressed in the primary tumor and corresponding hepatic as well as nodal metastases of patients with CRC. This context-sensitive diverse expression of Wnt-effector proteins may be important for future individualized targeted therapies.

\section{Introduction}

The wingless-type mouse mammary tumor virus intergration site family (Wnt) signaling pathway plays a major role in modulating cellular processes involved in development, differentiation and tissue homeostasis $(1,2)$. Interestingly, an aberrant Wnt-signaling pathway is generally implicated in cancer and other disease states, including the development of colorectal cancer (CRC) $(1,2)$. In fact, Wnt-signaling effectors regulate different processes that are essential for cancer progression, including tumor initiation and growth as well as differentiation and metastasis (1-3).

In the center of the Wnt signaling pathway is the flexible $\beta$-catenin which is tightly controlled mainly by Wnt- 1 , that stabilizes free pools of $\beta$-catenin and activates $\beta$-catenin-dependent transcription (4). Nuclear accumulation of $\beta$-catenin, is believed to be a crucial step in the carcinogenesis of CRC (1-3). Besides Wnt-1, the adenomatous polyposis coli (APC) gene is another important effector of the Wnt signaling pathway, and evidence suggest that APC is a negative regulator of $\beta$-catenin stability $(5,6)$. Of note, APC and $\beta$-catenin are frequently mutated in patients with CRC and overexpression of $\beta$-catenin or loss of APC function can lead to the development of CRC $(5,6)$. 
The Wnt signaling pathway is also involved in the metastatic progression of CRC (1-3). Activation of the pathway in the tumor itself frequently maintains a transcriptional course that is reminiscent of an epithelial-mesenchymal transition (EMT) which can provide cell migration and invasiveness (2). Additionally to the induction of EMT, $\beta$-catenin also modulates the expression of other factors that are important for metastatic progression, particularly matrix metalloproteinases (MMPs) and other factors that are essential for the regulation of the extracellular matrix (2). Finally, effectors of the Wnt signaling pathway directly control changes in cell morphology or signaling that are important for migration and invasion (2).

However, little is known regarding the exact interaction and context-sensitive expression of Wnt pathway effectors in the primary tumor and corresponding metastasis. Therefore, this study assessed the expression of the three most important effectors of the Wnt pathway, $\beta$-catenin, APC and Wnt-1, in the primary tumor and corresponding metastasis of patients with CRC.

\section{Patients and methods}

Twenty-four patients with metastatic CRC were included in this study. All study patients underwent surgical resection between 1996 and 2005 at the Surgical Department of the University of Düsseldorf (Düsseldorf, Germany). The formalin-fixed, paraffin-embedded tissue samples of the primary tumors and their corresponding liver metastases as well as 20 synchronous lymph node metastases were analyzed immunohistochemically. The pathological tumor stage and disease grades were classified according to the seventh edition of the tumor, node and metastasis classification of the International Union against Cancer (Geneva, Switzerland). The retrospective study regarding the immunohistochemical analyses on formalin-fixed, paraffin-embedded tissue samples was performed with approval by the ethics committee of the Medical Faculty of the University of Düsseldorf.

Tissue samples. Serial sections ( $4 \mu \mathrm{m}$ thick) were deparaffinised in xylene (Z.E.U.S. GmbH, Soltau, Germany) and dehydrated in a decreasing alcohol gradient, then incubated with different primary rabbit antibodies for $\beta$-catenin, APC and Wnt-1 (Thermo Scientific, Fremont, CA, USA). For immunohistochemistry, the $\mathrm{ABC}$ procedure (Vectastain ${ }^{\circledR} \mathrm{ABC}$ kit, Vector Laboratories, Inc., Burlingame, CA, USA) was used. Firstly, the slides were heated in a microwave oven in a Target Retrieval solution (DakoCytomation GmbH, Hamburg, Germany) at $95^{\circ} \mathrm{C}$ for 20 min prior to immunostaining. Subsequently, endogenous peroxidase activity was quenched in $0.3 \%$ hydrogen peroxide for $30 \mathrm{~min}$ (Paul W. Beyvers $\mathrm{GmbH}$, Berlin, Germa. Non-specific antibody binding was blocked with $10 \%$ normal serum (Vector Laboratories, Inc.) at room temperature. This was followed by incubating each section with the primary antibody at the favored concentrations for $30 \mathrm{~min}$ (rabbit monoclonal anti- $\beta$-catenin at 1:250 dilution; clone E247, APC and Wnt-1 (at 1:50 dilution; all rabbit polyclonal). Subsequent to washing with phosphate-buffered saline (PBS), the slides were incubated with $10 \%$ biotinylated secondary antibody for $30 \mathrm{~min}$ at room temperature, and finally incubated with $\mathrm{ABC}$ reagent for $30 \mathrm{~min}$. The immunoperoxidase
Table I. Clinicopathological characteristics of the study patients $(n=24)$.

\begin{tabular}{|c|c|}
\hline Patient characteristics & $\mathrm{n}(\%)$ \\
\hline \multicolumn{2}{|l|}{ Gender } \\
\hline Male & $13(54.16)$ \\
\hline Female & $11(45.83)$ \\
\hline \multicolumn{2}{|l|}{ Tumor location } \\
\hline Ascending colon & $7(29.16)$ \\
\hline Sigma & $6(25)$ \\
\hline Rectum & $6(25)$ \\
\hline Cecum & $4(16.6)$ \\
\hline Descending colon & $1(4.16)$ \\
\hline \multicolumn{2}{|l|}{ Tumor differentiation } \\
\hline G1 & 0 \\
\hline G2 & $17(70.83)$ \\
\hline G3 & $6(25)$ \\
\hline G4 & $1(4.16)$ \\
\hline \multicolumn{2}{|l|}{ T-stage } \\
\hline pT1 & $2(8.3)$ \\
\hline pT2 & $1(4.16)$ \\
\hline pT3 & $13(54.16)$ \\
\hline pT4 & $8(33.3)$ \\
\hline \multicolumn{2}{|l|}{ N-stage } \\
\hline pNO & $4(16.6)$ \\
\hline $\mathrm{pN} 1$ & $1(4.16)$ \\
\hline $\mathrm{pN} 2$ & $19(79.16)$ \\
\hline \multicolumn{2}{|l|}{ M-Stage } \\
\hline $\mathrm{pM} 0$ & $10(41.6)$ \\
\hline pM1 (HEP) & $14(58.3)$ \\
\hline \multicolumn{2}{|l|}{ R-stage } \\
\hline R0 & $19(79.16)$ \\
\hline R1 & $5(20.83)$ \\
\hline \multicolumn{2}{|c|}{ UICC-classification (stage) } \\
\hline I & $3(12.5)$ \\
\hline II & $1(4.16)$ \\
\hline III & $6(25)$ \\
\hline IV & $14(58.3)$ \\
\hline
\end{tabular}

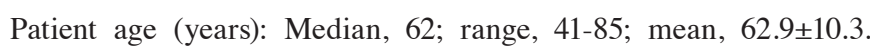
UICC, International Union against Cancer; G, grade; T, tumor; $p$, post-histopathological examination; $\mathrm{N}$, node, $\mathrm{M}$, metastasis; $\mathrm{R}$, resection; HEP, liver.

reaction was carried out with diaminobenzidine and nuclear counterstaining was carried out using Mayer's hematoxylin (Sigma-Aldrich, St. Louis, MI, USA). Membrane expression in the normal adjacent colonic epithelium was used as a positive control for each antibody, and sections from each block were incubated without the primary antibody as a negative control.

Serial sections were used to analyse the expression of $\beta$-catenin, APC and Wnt- 1 . In the primary tumors, central areas and the invasion front were analyzed separately. The percentage of tumor cells showing a positive staining was 

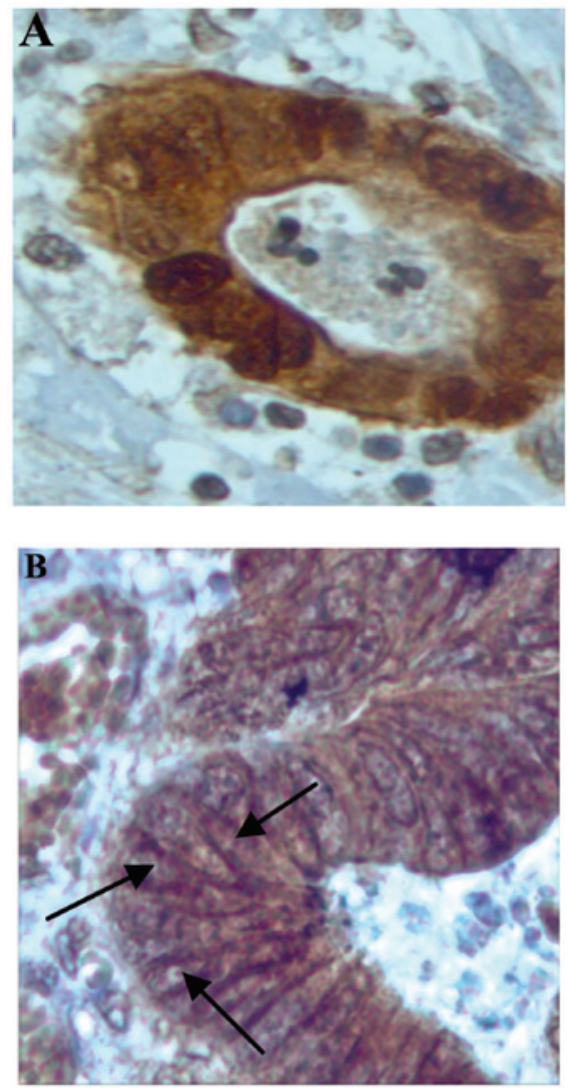

Figure 1. $\beta$-catenin expression shown by staining. (A) Nuclear and perinuclear cytoplasmic expression (magnification, x400). (B) Membranous expression (arrows) (magnification, $\mathrm{x} 200$ ).

scored and rounded up or down to the nearest $10 \%$ in 10 steps. The mean intensity of staining was scored between 0 and +3 ( 0 , no staining; +1 , weak; +2 , moderate; and +3 strong). This was performed according to the procedure to attain the immunoreactive score (IRS). Based on the percentage of positive tumor cells, four classes were defined $(<10,11-50$, 51-80, and $>80 \%$ ), while the mean staining intensity was separately evaluated as belonging to one of three classes (weak, moderate and strong). By multiplying the score values, an IRS scale ranging between 1 and 12 was obtained.

Statistical analysis. All statistical analyses were performed using SPSS 16.0 software (SPSS, Inc., Chicago, IL, USA). Correlations between parameters and the resulting P-values were calculated by applying the non-parametric Mann-Whitney $\mathrm{U}$ test or the Fisher's exact test. $\mathrm{P}<0.05$ was considered to indicate a statistically significant difference.

\section{Results}

Patients. Table I shows the clinicopathological characteristics of the study patients.

Nuclear $\beta$-catenin protein expression. There was a strong nuclear expression of $\beta$-catenin in $78.34 \%$ of the primary tumors, predominantly localized at the invasion front (Figs. 1A, 2 and 3). By contrast, cells in the tumor center often showed lower levels of nuclear staining $(49.17 \%, \mathrm{P}<0.0001)$. The normal

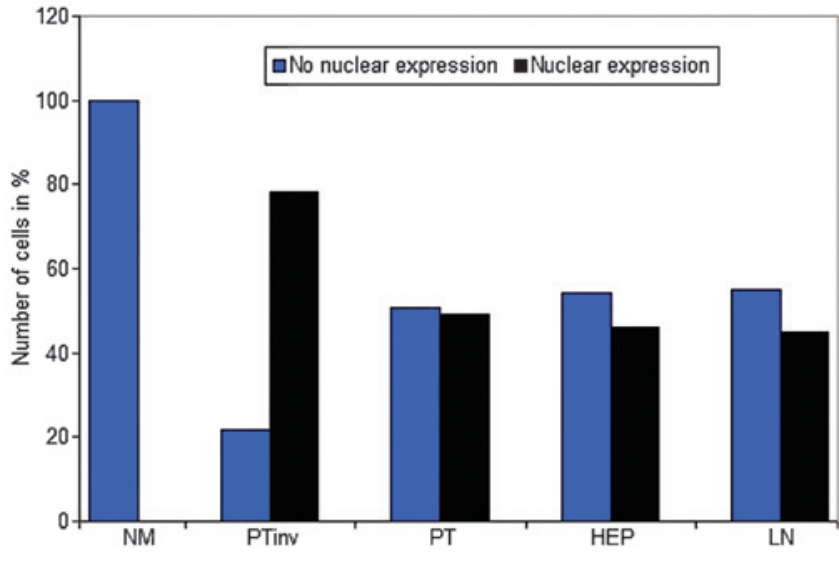

Figure 2. Nuclear $\beta$-catenin expression in the NM, PTinv, PT, HEP and LN metastasis. Nuclear expression at the PTinv is significantly increased $(\mathrm{P}<0.001)$. NM, normal colonic epithelium; PTinv, primary tumor invasion front; PT, primary tumor central area; HEP, liver; LN, lymph node.

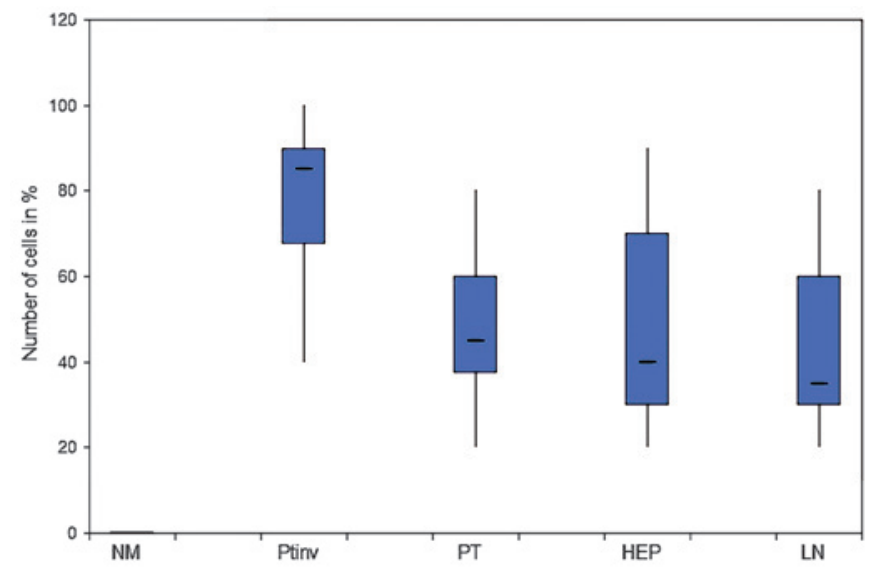

Figure 3. Expression patterns of nuclear $\beta$-catenin. Homogenous expression pattern in the PT and HEP as well as LN metastasis. There was a statistically significantly higher expression at the PTinv $(\mathrm{P}<0.001)$. NM, normal colonic epithelium; PTinv, primary tumor invasion front; PT, primary tumor central area; HEP, liver; LN, lymph node.

colonic epithelium exhibited no nuclear staining. In liver (45.84\%) and lymph node (45\%) metastases, the expression pattern was homogeneously associated with the tumor center.

Cytoplasmic $\beta$-catenin protein expression. In $74.5 \%$ of the primary tumors, $71.67 \%$ of the liver and $62 \%$ of the lymph node metastases, cytoplasmic $\beta$-catenin expression was strong (Table II). The homogenous expression pattern in primary tumors and metastatic lesions were statistically significant compared with the normal colon epithelium.

Membranous $\beta$-catenin protein expression. As shown in Table III, membranous $\beta$-catenin expression was almost homogenous in the normal colonic epithelium, primary tumor and metastatic lesions without any statistical significance (Fig. 1B and Table III). No significant associations were identified between $\beta$-catenin expression patterns and clinicopathological factors.

Wnt-1 protein expression. In $25.72 \%$ of the normal colonic epithelium and $20.52 \%$ of the primary tumors, tumor cells 
Table II. Cytoplasmic $\beta$-catenin expression.

\begin{tabular}{lccccr}
\hline & \multicolumn{4}{c}{ Staining intensity score (\% cells) } \\
\cline { 2 - 5 } Location of cells & 0 & $1+$ & $2+$ & $3+$ & P-value \\
\hline Normal epithelium & 0.00 & 8.75 & 49.58 & 41.67 & $<0.001$ \\
Primary tumor & 0.42 & 3.75 & 21.25 & 74.58 & 0.001 \\
Liver metastases & 0.00 & 2.92 & 25.42 & 71.67 & 0.017 \\
Lymph node metastases & 0.00 & 4.50 & 33.50 & 62.00 & \\
\hline
\end{tabular}

Table III. Membranous $\beta$-catenin expression.

\begin{tabular}{lcccr}
\hline & \multicolumn{4}{c}{ Staining intensity score (\% cells) } \\
\cline { 2 - 5 } Location of cells & 0 & $1+$ & $2+$ & $3+$ \\
\hline Normal epithelium & 0.00 & 1.25 & 22.08 & 76.67 \\
Primary tumor & 0.83 & 2.08 & 24.17 & 72.92 \\
Liver metastases & 0.00 & 0.00 & 18.75 & 81.25 \\
Lymph node metastases & 0.00 & 0.00 & 16.50 & 83.50 \\
\hline
\end{tabular}

Table IV. Wingless-type mouse mammary tumor virus integration site family, member 1 expression.

\begin{tabular}{lrccrr}
\hline & \multicolumn{4}{c}{ Staining intensity score (\% cells) } \\
\cline { 2 - 5 } Location of cells & \multicolumn{1}{c}{0} & $1+$ & $2+$ & $3+$ & P-value \\
\hline Normal epithelium & 5.20 & 28.04 & 37.68 & 25.72 \\
Primary tumor & 5.60 & 29.64 & 40.48 & 20.52 & 0.003 \\
Liver metastases & 20.80 & 55.64 & 16.48 & 3.32 & 11.09 \\
Lymph node metastases & 4.23 & 33.86 & 46.77 & \\
\hline
\end{tabular}

Table V. Adenomatous polyposis coli expression.

Staining intensity score (\% cells)

\begin{tabular}{lccccr}
\cline { 2 - 4 } Location of cells & 0 & $1+$ & $2+$ & $3+$ & P-value \\
\hline Normal epithelium & 19.17 & 44.58 & 35.42 & 0.83 & \\
Primary tumor & 24.17 & 65.42 & 10.42 & 0.00 & 0.022 \\
Liver metastases & 59.17 & 39.17 & 1.67 & 0.00 & 0.006 \\
Lymph node metastases & 35.00 & 59.50 & 5.50 & 0.00 & 0.012 \\
\hline
\end{tabular}

showed a strong staining (3+; Table IV). In liver metastases, expression of Wnt-1 (3.32\%) was significantly reduced $(\mathrm{P}=0.003)$ compared with the normal colonic epithelium.

APC protein expression. The APC expression patterns were homogenous in the primary tumor and metastatic lesions with no statistically significant differences. The strongest staining $(35.42 \%)$ was detected in the normal colonic epithelium which was significantly higher compared with the primary tumors $(\mathrm{P}=0.022)$, liver $(\mathrm{P}=0.006)$ and lymph node $(\mathrm{P}=0.012)$ metastases (Table V).

\section{Discussion}

By assessing the protein expression of the three most important effectors of the Wnt pathway, $\beta$-catenin, APC and Wnt-1, 
the present study was able to demonstrate that these major Wnt-effectors are heterogeneously expressed in the primary tumor and corresponding hepatic as well as nodal metastases of patients with CRC. This context-sensitive diverse expression of Wnt-effector proteins may be important for future individualized targeted therapies. In the center of the Wnt signaling pathway is the flexible $\beta$-catenin. Nuclear accumulation of $\beta$-catenin is believed to be a crucial step in the carcinogenesis of CRC (1-3).In fact, $\leq 80 \%$ of colorectal tumors exhibit nuclear accumulation of $\beta$-catenin (7-9). Furthermore, in $\leq 90 \%$ of all CRCs, a mutation in a key regulator of the Wnt signaling pathway, such as $\beta$-catenin, can be detected (10). The present results confirm that primary colorectal tumors present a high nuclear staining of $\beta$-catenin, whereas no relevant staining is found in normal colonic mucosa. In further detail, the present study revealed $\beta$-catenin to exhibit significantly higher expression at the tumor invasion front than in the tumor center, while in liver and lymph node metastases, the expression pattern was homogeneously associated with the tumor center. Similar results were reported by Brabletz et al (11) using immunohistochemistry in the analysis of CRC. This study group showed that the distribution of overexpressed $\beta$-catenin was not homogeneous, but a strong nuclear expression of $\beta$-catenin was predominantly localized at the invasion front, while in the tumor center often no nuclear staining was detected. Based on these results it can be hypothesized that due to the strong activation of the Wnt signaling pathway, an EMT of the tumor cells is induced which enables them to have a high metastatic potential (12). In addition, the relatively reduced staining of $\beta$-catenin in the liver and lymph node metastases suggests that during the metastatic process, tumor cells run through a mesenchymal-epithelial transition. Brabletz et al (10) also demonstrated in colorectal metastases a reduced $\beta$-catenin expression compared with the invasion front of the primary tumor. Therefore, cancer dissemination appears to be a dynamic process where tumor cells are interacting at different 'Wnt activity levels' with each other (13).

Wnt-1, a glycoprotein that associates with cell membranes and likely functions as a key regulator of cellular adhesions, is another important factor of the Wnt signaling pathway which stabilizes free pools of $\beta$-catenin and activates $\beta$-catenin-dependent transcription (14). Of note, the present study was not able to show an overexpression of Wnt-1 in tumor tissue compared with normal colonic mucosa. In fact, Wnt-1 expression was actually significantly reduced in liver metastases. Even the current literature shows conflicting results regarding the expression profiles of Wnt-1 in CRC. For example, Stanczak et al (15) determined the expression and localization of E-cadherin, $\beta$-catenin and Wnt-1 proteins in advanced CRC immunohistochemically. They demonstrated a strong Wnt-1 staining in the normal colonic epithelium, while in the majority of primary tumors the expression was decreased. By contrast, Khor et al (16) revealed in 47 colorectal tissue samples that intratumoral protein expression of Wnt-1 was significantly increased in the primary tumor compared with the normal epithelium. Finally, Holcombe et al (17) revealed in CRC that Wnt-1 is expressed equally and strongly in normal and malignant colon tissues. Currently, only speculation is possible as to the reasons for these discrepant findings. Possible reasons are: i) There are Wnt-1 independent mechanisms that increase the nuclear accumulation of $\beta$-catenin, such as APC mutation; ii) besides the canonical $\mathrm{Wnt} / \beta$-catenin pathway, other non-canonical Wnt pathways have been described which are $\beta$-catenin and Wnt-1 independent, and appear also to play an important role in cell adhesion and cell migration (18).

The present study results demonstrate that the highest APC protein expression was detected in the normal colonic mucosa, whereas the expression in the primary tumors and particularly in the metastases was significantly lower. These findings are partially in agreement with a study by Chen et al (19) that performed a molecular analysis of APC in 39 primary CRC and 24 liver metastases samples. While this previous study also showed a decreased APC expression in primary tumor tissues compared with normal colonic mucosa, the expression in the liver metastases remained strong. The reduced APC expression in the primary tumors may be explained by the following reasons: i) Due to the high rate of APC mutations in CRC, the APC gene is generally inactivated in this type of cancer and consequently its protein expression is decreased; ii) APC inactivation can also emerge due to promoter methylation which is frequently described in CRC (20).

In conclusion, the present study results demonstrate heterogeneously expressed major Wnt effectors in the primary tumor and corresponding hepatic as well as nodal metastases of patients with CRC. Similar findings were reported by Wu et al (21) comparing the therapeutic target expression and promoter methylation between primary breast tumors and their multifocal metastases. Notably, the study showed that therapeutic targets identified in the primary breast tumors do not reflect targets present in the metastatic sites. Therefore, the data about heterogenous expression in the primary tumor and corresponding metastasis is an important aspect regarding the accepted linear progression model which assumed similar molecular events in the primary tumor and metastasis. In fact, in this model tumor development is based on a stepwise progression from an early pattern to invasive and finally metastatic cancer (22). On the contrary, the present and previous studies support the model of parallel progression (23). Dissemination of single tumor cells is an early step in the metastatic process and leads to an allopatric selection of variant tumor cells, adjusted to their specific microenvironment (24). Therefore, the parallel progression model predicts greater disparity between metastatic lesions and primary tumor cells than does the linear progression model.

\section{References}

1. Klaus A and Birchmeier W: Wnt signalling and its impact on development and cancer. Nat Rev Caner 8: 387-398, 2008.

2. Anastas JN and Moon RT: WNT signalling pathways as therapeutic targets in cancer. Nat Rev Caner 13: 11-26, 2013.

3. White BD, Chien AJ and Dawson DW: Dysregulation of Wnt $/ \beta$-catenin signalling in gastrointestinal cancers. Gastroenterology 142: 219-232, 2012.

4. Papkoff J, Rubinfeld B, Schryver B and Polakis P: Wnt-1 regulates free pools of catenins and stabilizes APC-catenin complexes. Mol Cell Biol 16: 2128-2134, 1996.

5. Korinek V, Barker N, Morin PJ, et al: Constitutive transcriptional activation by a $\beta$-catenin-Tcf complex in APC-/-colon carcinoma. Science 275: 1784-1787, 1997.

6. Morin PJ, Sparks AB, Korinek V, et al: Activation of $\beta$-catenin-Tcf signaling in colon cancer by mutations in $\beta$-catenin or APC. Science 275: 1787-1790, 1997. 
7. Martensson A, Oberg A, Jung A, et al: Beta-catenin expression in relation to genetic instability and prognosis in colorectal cancer. Oncol Rep 17: 447-452, 2007.

8. Wanitsuwan W, Kanngurn S, Boonpipattanapong T, et al: Overall expression of beta-catenin outperforms its nuclear accumulation in predicting outcomes of colorectal cancers. World $\mathrm{J}$ Gastroenterol 14: 6052-6059, 2008.

9. Elzagheid A, Buhmeida A, Korkeila E, et al: Nuclear beta-catenin expression as a prognostic factor in advanced colorectal carcinoma. World J Gastroenterol 14: 3866-3871, 2008.

10. Brabletz T, Jung A, Reu S, et al: Variable beta-catenin expression in colorectal cancers indicates tumor progression driven by the tumor environment. Proc Natl Acad Sci USA 98: 10356-10361, 2001.

11. Brabletz T, Jung A, Hermann K, Günther K, Hohenberger W and Kirchner T: Nuclear overexpression of the oncoprotein beta-catenin in colorectal cancer is localized predominantly at the invasion front. Pathol Res Pract 194: 701-704, 1998.

12. Gabbert H, Wagner R, Moll R and Gerharz CD: Tumor dedifferentiation: an important step in tumor invasion. Clin Exp Metastasis 3: 257-279, 1985.

13. Hlubek F, Brabletz T, Budczies J, Pfeiffer S, Jung A and Kirchner T: Heterogeneous expression of Wnt/beta-catenin target genes within colorectal cancer. Int J Cancer 121: 1941-1948, 2007.

14. Behrens J: The role of Wnt signalling pathway in colorectal tumorigenesis. Biochem Soc Trans 33: 672-675, 2005.
15. Stanczak A, Stec R, Bodnar L, et al: Prognostic significance of Wnt-1, beta-Catenin and E-Cadherin expression in advanced colorectal carcinoma. Pathol Oncol Res 17: 955-963, 2011.

16. Khor TO, Gul YA, Ithnin H and Seow HF: A comparative study of expression of Wnt-1, WISP-1, survivin and cyclin-D1 in colorectal carcinoma. Int J Colorectal Dis 21: 291-300, 2006.

17. Holcombe RF, Marsh JL, Waterman ML, Lin F, et al: Expression of Wnt ligands and Frizzled receptors in colonic mucosa and in colon carcinoma. Mol Pathol 55: 220-226, 2002.

18. De A: Wnt/Ca2+ signaling pathway: a brief overview. Acta Biochim Biophys Shanghai 2011; 43: 745-756, 2011.

19. Chen J, Röcken C, Lofton-Day C, Schulz HU, et al: Molecular analysis of APC promoter methylation and protein expression in colorectal cancer metastasis. Carcinogenesis 26: 37-43, 2005.

20. Baylin SB and Herman JG: DNA hypermethylation in tumorigenesis: epigenetics joins genetics. Trends Genet 16: 168-174, 2000.

21. Wu JM,Fackler MJ,Halushka MK, Molavi DW, et al: Heterogeneity of breast cancer metastases: comparison of therapeutic target expression and promoter methylation between primary tumors and their multifocal metastases. Clin Cancer Res 14: 1938-1946, 2008.

22. Fearon ER and Vogelstein B: A genetic model for colorectal tumorigenesis. Cell 61: 759-767, 1990.

23. Collins VP, Loeffler RK and Tivey H: Observations on growth rates of human tumors. Am J Roentgenol Radium Ther Nucl Med 76: 988-1000, 1956.

24. Klein CA: Parallel progression of primary tumours and metastases. Nat Rev Cancer 9: 302-312, 2009. 